\title{
Every Fourier optical system is equivalent to consecutive fractional-Fourier-domain filtering
}

\author{
Haldun M. Ozaktas and David Mendlovic
}

\begin{abstract}
We consider optical systems composed of an arbitrary number of lenses and filters, separated by arbitrary distances, under the standard approximations of Fourier optics. We show that every such system is equivalent to (i) consecutive filtering operations in several fractional Fourier domains and (ii) consecutive filtering operations alternately in the space and the frequency domains.

Key words: Fourier optics, optical information processing, fractional Fourier transform. (c) 1996 Optical Society of America
\end{abstract}

In this paper we consider systems composed of an arbitrary number of lenses and filters-separated by arbitrary distances [Fig. 1(a)], under the standard approximations of Fourier optics. ${ }^{1}$ We show that every such system is equivalent to, and can be modeled as, both of the following: (1) Consecutive filtering operations in several fractional Fourier domains [Fig. 1(c)]. Each fractional-Fourier-transform stage transforms from one fractional domain to another in which a multiplicative filter is applied. More precisely, every Fourier optical system is equivalent to a sequence of appropriately chosen multiplicative filters inserted between fractional-Fourier-transform stages with appropriately chosen orders. (2) Consecutive filtering operations alternately in the space and the frequency domains [Fig. 1(d)]. Each time a Fourier transform is applied we alternate between the space and frequency domains, in which multiplicative filters are applied. More precisely, every first-order optical system is equivalent to a sequence of appropriately chosen multiplicative filters inserted between appropriately scaled Fouriertransform stages. These equivalences provide considerable conceptual simplification and insight regarding such systems and should also facilitate their design and analysis.

Members of the class of quadratic-phase systems ${ }^{2-4}$ are characterized by linear transformations

H. M. Ozaktas is with the Department of Electrical Engineering, Bilkent University, Bilkent 06533, Ankara, Turkey. D. Mendlovic is with the Faculty of Engineering, Tel-Aviv University, Tel Aviv 69978, Israel.

Received 8 August 1995.

0003-6935/96/173167-04\$10.00/0

(1) 1996 Optical Society of America of the form

$$
\begin{aligned}
p_{\text {out }}(x) & =\int_{-\infty}^{\infty} h\left(x, x^{\prime}\right) p_{\text {in }}\left(x^{\prime}\right) \mathrm{d} x^{\prime}, \\
h\left(x, x^{\prime}\right) & =C \exp \left[i \pi\left(\alpha x^{2}-2 \beta x x^{\prime}+\gamma x^{\prime 2}\right)\right],
\end{aligned}
$$

where $C$ is a complex constant, and $\alpha, \beta$, and $\gamma$ are real constants. Optical systems involving an arbitrary sequence of thin lenses separated by arbitrary sections of free space belong to this class. ${ }^{5}$ The class of Fourier optical systems (more precisely called first-order optical systems ${ }^{3}$ ) consists of arbitrary thin transmissive filters sandwiched between arbitrary quadratic-phase systems [Figs. 1(a) and 1(b)].

The kernels associated with a thin lens with focal length $f$, propagation over a distance $d$, and a multiplicative filter $h(x)$ are given respectively by ${ }^{1}$

$$
\begin{aligned}
h_{\text {lens }}\left(x, x^{\prime}\right) & =C_{\text {lens }} \delta\left(x-x^{\prime}\right) \exp \left(-i \pi x^{2} / \lambda f\right), \\
h_{\text {space }}\left(x, x^{\prime}\right) & =C_{\text {space }} \exp \left[i \pi\left(x-x^{\prime}\right)^{2} / \lambda d\right], \\
h_{\text {filter }}\left(x, x^{\prime}\right) & =\delta\left(x-x^{\prime}\right) h(x) .
\end{aligned}
$$

$\lambda$ is the wavelength of light. One-dimensional notation is employed for simplicity.

The fractional Fourier transform ${ }^{2-9}$ is a subclass of the class of quadratic-phase systems. In its most general form, the fractional Fourier transform has three parameters: the order $a$, the input scale parameter $s_{\text {in }}$, and the output scale parameter $s_{\text {out }}{ }^{2}$ (When we set $s_{\text {in }}=s_{\text {out }}=1$, we recover the pure mathematical form of the transform.) The kernel is 
a.

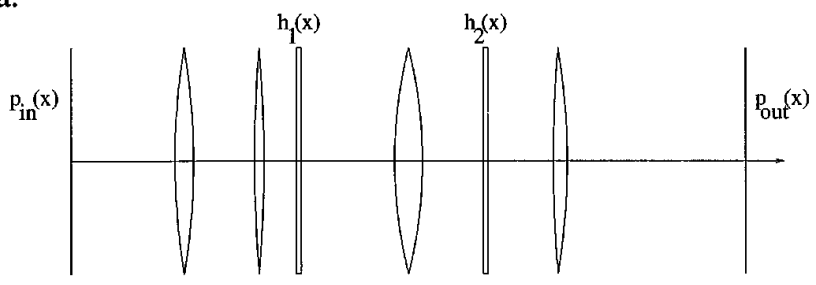

b.

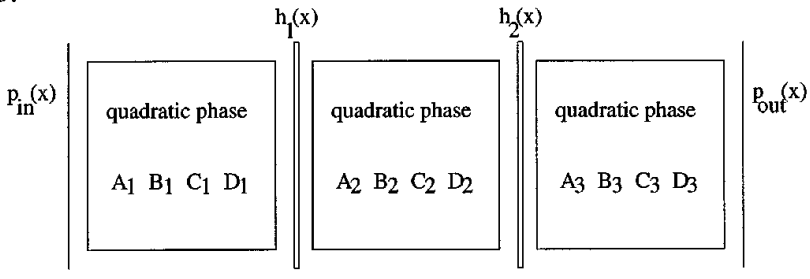

c.

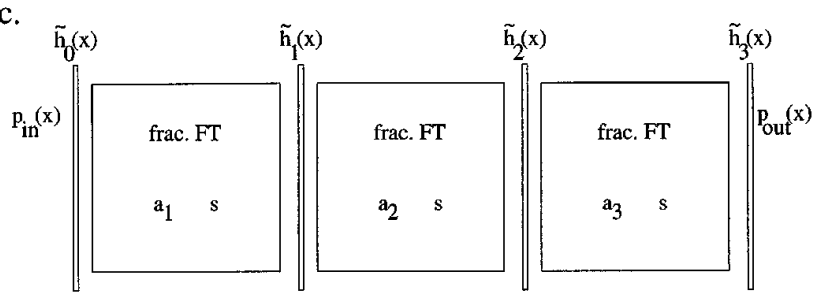

d.

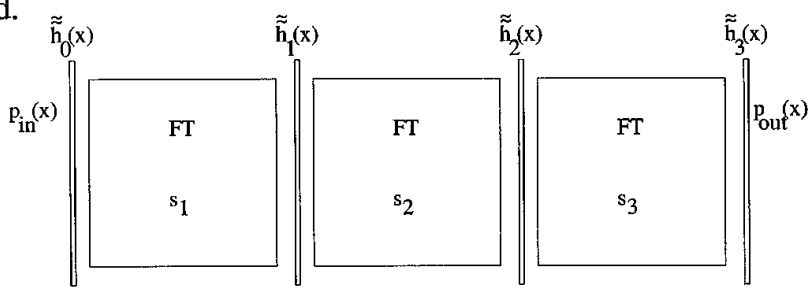

Fig. 1. a. First-order optical system with input $p_{\text {in }}(x)$ and output $p_{\text {out }}(x)$, consisting of several lenses, filters, and sections of free space. The transmittance functions of the filters are indicated directly above them. b. The system modeled as a sequence of multiplicative filters sandwiched between quadratic-phase systems, each of which is characterized by its matrix parameters $A_{j}$, $B_{j}, C_{j}$, and $D_{j}$. c. The system modeled as a sequence of multiplicative filters sandwiched between fractional-Fourier-transform stages, each of which is characterized by its order $a_{j}$. The scale parameter $s$ is the same for all stages. d. The system modeled as a sequence of multiplicative filters sandwiched between conventional Fourier-transform stages, each of which is characterized by its scale parameter $s_{j}$.

given by

$$
\begin{aligned}
& h_{\text {frac }}\left(x, x^{\prime}\right)= \\
& \quad C_{\text {frac }} \exp \left[i \pi\left(\cot \phi \frac{x^{2}}{s_{\text {out }}^{2}}-2 \csc \phi \frac{x x^{\prime}}{s_{\text {in }} s_{\text {out }}}+\cot \phi \frac{x^{\prime 2}}{s_{\text {in }}^{2}}\right)\right],
\end{aligned}
$$

where $\phi=a \pi / 2$. The ordinary Fourier transform is obtained when we set $a=1$. In this paper it is sufficient to employ fractional transforms with $s_{\text {in }}=$ $s_{\text {out }} \equiv s$.

Apart from the constant factor $C$, which has no effect on the resulting spatial distribution, ${ }^{10}$ a member of the class of quadratic-phase systems is completely specified by the three parameters $\alpha, \beta$, and $\gamma$ [Eq. (1)]. Alternatively, such a system can also be completely specified by the transformation matrix ${ }^{2,3,11-14}$

$$
\left[\begin{array}{ll}
A & B \\
C & D
\end{array}\right] \equiv\left[\begin{array}{cc}
\gamma / \beta & 1 / \beta \\
-\beta+\alpha \gamma / \beta & \alpha / \beta
\end{array}\right]
$$

with $A D-B C=1$. If several systems, each characterized by such a matrix are cascaded, the matrix characterizing the overall system can be found by multiplying the matrices of the several systems. ${ }^{2,3}$

First, we show that any quadratic-phase system can be expressed as the concatenation of a lens followed by a fractional Fourier transform followed by another lens, as expressed by ${ }^{2}$

$$
\begin{aligned}
{\left[\begin{array}{ll}
A & B \\
C & D
\end{array}\right]=} & {\left[\begin{array}{cc}
1 & 0 \\
-1 / f_{r} \lambda & 1
\end{array}\right]\left[\begin{array}{cc}
\cos \phi & s^{2} \sin \phi \\
-s^{-2} \sin \phi & \cos \phi
\end{array}\right] } \\
& \times\left[\begin{array}{cc}
1 & 0 \\
-1 / f_{l} \lambda & 1
\end{array}\right]
\end{aligned}
$$

where $f_{l}$ and $f_{r}$ are the focal lengths of the lenses on the left and the right of the fractional Fourier transformer, respectively. The matrices appearing on the right-hand side of the above equation are obtained by Eqs. (2), (5), and (6). (For other similar decompositions see, for instance, Refs. 11-14.)

Now, let us set the scale parameter $s$ according to our free choice and solve the above equation for $\phi, f_{l}$ and $f_{r}$, in terms of $A, B, C, D$, and $s$. The result is

$$
\begin{aligned}
\sin \phi & =\frac{B}{s^{2}}, \quad \phi \in[-\pi / 2, \pi / 2], \\
f_{l} \lambda & =\frac{B}{\sqrt{1-B^{2} / s^{4}}-A}, \quad f_{r} \lambda=\frac{B}{\sqrt{1-B^{2} / s^{4}}-D} .
\end{aligned}
$$

(Upon writing the above solution, we realize that we are not fully free in choosing $s$; we may choose it freely subject to the constraint $s^{2} \geq|B|$.)

The existence of the above solution implies that each of the quadratic-phase systems in Fig. 1(b) can be replaced by a lens followed by a fractional Fourier transform followed by a lens. Now, by absorbing the transmittance functions of the lenses in that of the adjacent filters, that is, by defining

$$
\tilde{h}_{j}(x)=\exp \left(\frac{-i \pi x^{2}}{f_{l_{(j+1)}} \lambda}\right) h_{j}(x) \exp \left(\frac{-i \pi x^{2}}{f_{r_{j}} \lambda}\right),
$$

we arrive at the configuration of Fig. 1(c), which is what we sought to show.

Returning to Eq. (7), let us now set $a=1(\phi=\pi / 2) .15$ This time, we can solve for $s, f_{l}$, and $f_{r}$ in terms of $A$, 
$B, C$, and $D$ as follows:

$$
s=\sqrt{B}, \quad f_{l} \lambda=\frac{-B}{A}, \quad f_{r} \lambda=\frac{-B}{D} .
$$

If $B<0$, the above solution will not be valid, but we can obtain a complementary and valid solution in the same manner by setting $a=-1$ (corresponding to an inverse Fourier transform) instead of $a=1$.

Again, each of the quadratic-phase systems in Fig. 1(b) can be replaced by a lens followed by a scaled Fourier transform followed by a lens. By causing the absorption of the transmittance functions of the lenses in that of adjacent filters as in Eq. (10), we also prove the second result.

Fractional Fourier transforms can be realized optically with bulk lenses ${ }^{2,16-18}$ or quadratic gradedindex media. ${ }^{19}$ Thus, for instance, our first result implies that any first-order optical system can be realized by sandwiching of multiplicative filters between segments of graded-index media. This would essentially be a physical embodiment of Fig. 1(c). The fractional Fourier transform also has an $O(N \log$ $N)$ time digital implementation. ${ }^{20,21}$

In previous work ${ }^{6,19}$ we had referred to filtering systems of the form of Fig. 1(c) as generalized filtering systems. In contrast to conventional Fourierdomain filtering systems these systems employ a multitude of filters in several consecutive fractional Fourier domains. Whereas the use of a single conventional Fourier-domain filter allows us to realize only space-invariant (convolution type) systems, generalized filtering systems represent a more general class of linear systems, including many spacevariant operations that are useful for a variety of applications such as the elimination of nonstationary noise and restoration of signals under spacevariant distortion models. ${ }^{21,22}$

Our first result means that the analysis or the design of Fourier optical filtering systems of arbitrary configuration [Fig. 1(a)] is equivalent to, and thus can be reduced to, that of a generalized filtering system. In other words, any arbitrary Fourier optical filtering system acts on the input in a way that is equivalent to applying consecutive filters in fractional Fourier domains. Any results, methods of analysis or design, and algorithms developed for generalized filtering systems are thus also applicable to such Fourier optical systems.

An important special case of the second result is also worth noting. Since a fractional Fourier transformer is a special type of quadratic-phase system, it can also be expressed in terms of a lens followed by an ordinary Fourier transform followed by a lens. Thus, according to our second result, any system of the form depicted in Fig. 1(c) can be reduced to one of the form depicted in Fig. 1(d). This means that generalized filtering systems employing fractional transforms [as in Fig. 1(c)] can be reduced to generalized filtering systems employing only the ordinary Fourier transform [as in Fig. 1(d)]. Applying multi- plicative filters alternately in the space and the Fourier domains allows us to do everything that we can do by applying filters in fractional domains. [The following generalization can also be demonstrated similarly: Applying multiplicative filters alternately in any given two domains (provided their orders do not differ by an integer multiple of 2 ) allows us to do everything that can be done by any of the configurations mentioned in this paper.]

This result does not compromise the conceptual and practical utility of the fractional Fourier transform. The fractional transform may be conceptually indispensable in devising an algorithm or designing an effective filter, ${ }^{22}$ even if the system is then reduced to one that does not employ fractional Fourier transforms. Furthermore, one would not necessarily engage in such a reduction, since computation of the fractional transform-both optically and digitally-is not more difficult than computation of the ordinary transform. The computation of ordinary and fractional transforms can both be reduced to each other. The implementation of Fig. 1(c) is not more difficult than that of Fig. 1(d).

From a practical viewpoint the implementation of the necessary filters may be much easier in certain domains, as compared with others. For instance, in chirp elimination ${ }^{23}$ the filters necessary in fractional domains are simple apertures or knife edges, whereas in the ordinary space and Fourier domains they would have to be complex functions. Furthermore, it may be easier to minimize deviations from the standard approximations of Fourier optics in one configuration as opposed to another. In conclusion, the equivalence results shown in this paper should be used to increase the number of alternative physical realizations that are nominally (within the approximations of Fourier optics) equivalent, not to reduce them to one. These alternative realizations provide additional degrees of freedom that may allow us to deal effectively with certain practical and technical constraints, such as the need to use catalog optics, the need to limit sensitivity to parameter deviations, and limitations on the realizability of filters.

We conclude by briefly outlining an alternative formulation of our first result. An easy generalization of Eq. (7) to the case $s_{\text {in }} \neq s_{\text {out }}$ has the following implication: Any quadratic-phase system can be interpreted as a fractional Fourier transform by choice of appropriate input and output scale factors and spherical reference surfaces. ${ }^{2}$ Since there are sufficient degrees of freedom, we are allowed to fix the scale factor and the radius of the reference surface on the input side. Once this is done, the order of the transform, and the scale factor and the radius of the reference surface on the output side, are determined in terms of $A, B, C$, and $D$. By introducing the constraint that the input scale factor and the radius of the input reference surface of each stage be set equal to the corresponding output quantities of the previous stage, we can reduce a 
system of the form depicted in Fig. 1(b) to a system of the form depicted in Fig. $1(\mathrm{c})$ such that $h_{j}(x)=\tilde{h}_{j}(x)$. When the radii of the spherical reference surfaces on both sides of the filter are the same, we simply find the amplitude distribution on the right by multiplying the amplitude distribution on the left by $h_{j}(x)$.] Unlike in the original derivation above, in this system the scale factor is not uniform throughout the stages.

\section{References and Notes}

1. B. E. A. Saleh and M. C. Teich, Fundamentals of Photonics (Wiley, New York, 1991).

2. H. M. Ozaktas and D. Mendlovic, "Fractional Fourier Optics," J. Opt. Soc. Am. A 12, 743-751 (1995).

3. M. J. Bastiaans, "Wigner distribution function and its application to first-order optics," J. Opt. Soc. Am. A 69, 1710-1716 (1979).

4. K. B. Wolf, Integral Transforms in Science and Engineering (Plenum, New York, 1979).

5. Quadratic graded-index media also belong to this class, and our results remain valid also for systems containing arbitrary sections of such media.

6. H. M. Ozaktas, B. Barshan, D. Mendlovic, and L. Onural, "Convolution, filtering, and multiplexing in fractional Fourier domains and their relation to chirp and wavelet transforms," J. Opt. Soc. Am. A 11, 547-559 (1994).

7. A. C. McBride and F. H. Kerr, "On Namia's fractional Fourier transform,” IMA J. Appl. Math. 39, 159-175 (1987).

8. L. B. Almeida, "The fractional Fourier transform and timefrequency representations," IEEE Trans. Acoust. Speech Signal Process. 42, 3084-3091 (1994).

9. H. M. Ozaktas and D. Mendlovic, "Fractional Fourier transform as a tool for analyzing beam propagation and spherical mirror resonators," Opt. Lett. 19, 1678-1680 (1994).

10. Although the factor $C$ has no effect on the resulting spatial distribution, it is not arbitrary, and its magnitude is determined by the requirement of power conservation for a unitary system. ${ }^{11}$
11. S. Abe and J. T. Sheridan, "Optical operations on wave functions as the Abelian subgroups of the special affine Fourier transformation," Opt. Lett. 19, 1801-1803 (1994).

12. M. Nazarathy and J. Shamir, "First-order optics—a canonical operator representation: lossless systems," J. Opt. Soc. Am. 72, 356-364 (1982).

13. A. Papoulis, Signal Analysis (McGraw-Hill, New York, 1977).

14. A. Siegman, Lasers (University Science, Mill Valley, Calif., 1986).

15. We can find similar results by setting $a$ to any other value as well. The conventional Fourier transform is no more privileged than fractional transforms of other orders.

16. A. W. Lohmann, "Image rotation, Wigner rotation, and the fractional Fourier transform," J. Opt. Soc. Am. A 10, 21812186 (1993).

17. L. M. Bernardo and O. D. D. Soares, "Fractional Fourier transforms and imaging," J. Opt. Soc. Am. A 11, 2622-2626 (1994).

18. P. Pellat-Finet and G. Bonnet, "Fractional order Fourier transform and Fourier optics," Opt. Commun. 111, 141-154 (1994).

19. H. M. Ozaktas and D. Mendlovic, "Fractional Fourier transforms and their optical implementations. II," J. Opt. Soc. Am. A 10, 2522-2531 (1993).

20. H. M. Ozaktas, O. Arikan, M. A. Kutay, and G. Bozdagi, "Digital computation of the fractional Fourier transform," IEEE Trans. Signal Process. (to be published).

21. M. A. Kutay, H. M. Ozaktas, O. Arikan, and L. Onural, "Optimal filtering in fractional Fourier domains," IEEE Trans. Acoust. Speech Signal Process. (to be published).

22. Convincing examples of the utility of the fractional Fourier transform in designing filtering systems may be found in Refs. 6, 21, and 23, and in D. Mendlovic, H. M. Ozaktas, and A. W. Lohmann, "Fractional correlation," Appl. Opt. 34, 303-309 (1995).

23. H. M. Ozaktas, B. Barshan, and D. Mendlovic, "Convolution and filtering in fractional Fourier domains," Opt. Rev. 1, 15-16 (1994); R. G. Dorsch, A. W. Lohmann, Y. Bitran, D. Mendlovic, and H. M. Ozaktas, "Chirp filtering in the fractional Fourier domain,” Appl. Opt. 33, 7599-7602 (1994). 